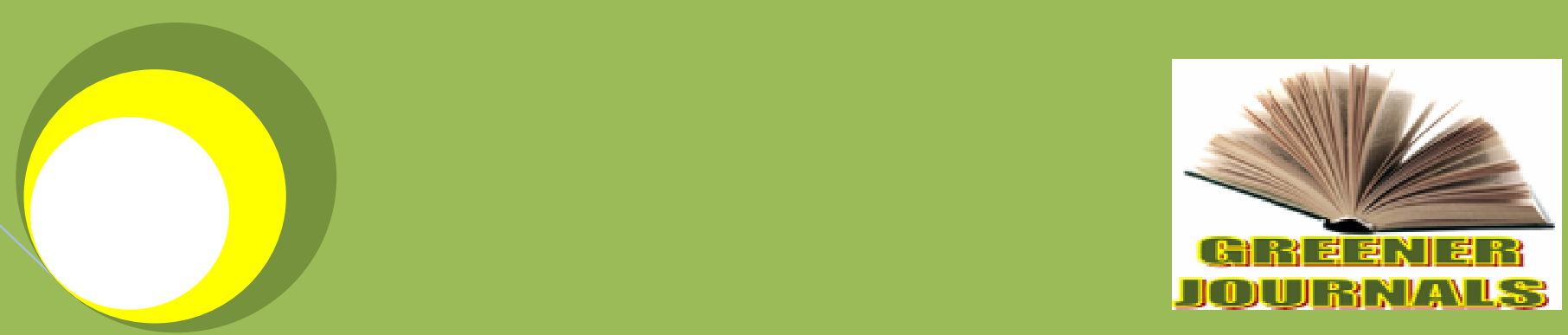

Greener. outrnal of educational Research

ISSN: 2276-7789 ICV: $\mathbf{6 . 0 5}$

Submitted: $23 / 05 / 2016$

Accepted: $27 / 05 / 2016$

Published: 31/05/2016

DOI: http://doi.org/10.15580/GJER.2016.3.052316095

Influence of Mental

Harassment Ban on

Student Discipline in

Secondary Schools in

Kenya: A Case Study

of Ugenya, Gem and

Siaya Sub-Counties

By

David Otieno Onyango

Enose M.W. Simatwa

Julius O. Gogo 


\title{
Influence of Mental Harassment Ban on Student Discipline in Secondary Schools in Kenya: A Case Study of Ugenya, Gem and Siaya Sub-Counties
}

\section{David Otieno Onyango, Enose M.W. Simatwa*, Julius O. Gogo}

\author{
Department of Educational Management and Foundations, Maseno University
}

${ }^{*}$ Corresponding Author's Email: simatwae@ yahoo. com

\begin{abstract}
The Kenya government banned Mental Harassment in schools as stipulated in The Basic Education Act, 2013. This was as a result of the recommendations of the Task Force on Student Discipline and Unrests in Secondary Schools, which revealed that Mental Harassment was one of the major contributors to indiscipline among students. Despite the ban, the level of indiscipline in schools had remained a major concern in Ugenya, Gem and Siaya sub-counties, where cases of indiscipline for the years 2010 - 2015 were $514(51 \%)$ higher than those experienced in Siaya County, $694(44 \%)$ and national, $51,600(42.7 \%)$ for the same period. It is important to note that mental harassment is one of the strategies still used in managing students despite the ban and the fact that it was found wanting and counterproductive at a time when discipline cases among students had hit the ceiling; in the early part of this $21^{\text {st }}$ Century. The high level of indiscipline had been characterized by students' threats against school authorities, arson, vandalism, physical attack on teachers, unrests, strikes, complaints of high-handedness, drug abuse, sexual harassment and terrorist threats. All these infractions were mainly targeted at teachers, school administrators and school prefects. Mental harassment as a strategy of managing student discipline involves: reprimanding, use of abusive and belittling language, sarcasm, sexual harassment, name calling, shouting, verbal warnings, insults, detention, withdrawal of privileges, scolding and unwarranted criticisms that inflict psychological pain or leads to psychological torture. Mental harassment is mainly used to curb infractions like absenteeism, truancy, failure to do homework, lateness, laziness, poor academic performance, insubordination, non-adherence to dress code, noisemaking, lack of participation in class work and disobedience. Mental harassment was outlawed through enactment of the Basic Education Act 2013, The Constitution of Kenya 2010 and The Children Act 2001 based on reports on school indiscipline that had identified mental harassment as one of the major causes of students' indiscipline. The study established that there was a strong, positive and significant relationship between the level of mental harassment ban and students' discipline. The level of mental harassment ban was $60 \%$, while the level of students discipline was $40 \%$. Mental harassment ban accounted for $68.5 \%$ of the variation in students' level of discipline. Regression analysis revealed that the increase in mental harassment ban increased student discipline.
\end{abstract}

Key Words: Influence, Mental Harassment Ban, Student Discipline, public Secondary Schools, Ugenya, Gem Siaya SubCounties, Kenya.

\section{INTRODUCTION}

Students discipline is critical in the attainment of positive school outcomes. This is because inter alia provides a sense of direction among learners besides increasing a teacher's job satisfaction which is a critical correlate of commitment to school goals (Simiyu, 2003). In spite of the crucial role that discipline plays in the overall school outcomes, the condition of students' discipline in Kenya's secondary schools has been disheartening. This is because hardly, a school term goes without incidence of violent behavior being reported in the mass media (Ogetange, 2012). Teachers use different methods to manage discipline in schools. One of the methods used and the most controversial is mental harassment. In Kenya the government enacted the Education Act, 2013, which banned mental harassment in schools.

The Education Act, 2013 does not give details of mental harassment. It is upon the individual teacher to interpret the Act. According to this study, mental harassment includes rebuking a student, insulting students, verbal reprimanding or any form of psychological torture. Alternative methods of student discipline management include guidance and counseling, time off, withdrawal of privileges and suspension. Teachers are of the opinion that these alternative methods of student discipline management are not effective as they take too much time (Busienei, 2012). 
The government emphasized on guidance and counseling, as an alternative to mental harassment. According to Kaburu (2006), the use of guidance and counseling to manage student discipline is not effective because teachers lack guidance and counseling skills. This method is also time consuming and schools lack resources for effective guidance and counseling programs. Although, the government has done a lot in order to curb violence and indiscipline in schools, there are still some cases of violence/strikes in schools. Furthermore, many cases of other forms of indiscipline have been reported in the mass media (Murithi, 2010). Schools are now experiencing many forms of indiscipline. Table 1 shows some forms of indiscipline cases experienced in Ugenya, Gem and Siaya SubCounties.

Table 1: Reported cases of indiscipline experienced in secondary school in Ugenya, Gem and Siaya SubCounties: 2010-2015

\begin{tabular}{lccccccc}
\hline Type of Indiscipline & $\mathbf{2 0 1 0}$ & $\mathbf{2 0 1 1}$ & $\mathbf{2 0 1 2}$ & $\mathbf{2 0 1 3}$ & $\mathbf{2 0 1 4}$ & $\mathbf{2 0 1 5}$ & Total \\
\hline Strikes & 15 & 14 & 13 & 10 & 10 & 9 & $\mathbf{7 1}$ \\
Bullying & 14 & 15 & 18 & 14 & 15 & 13 & $\mathbf{8 9}$ \\
Drugs & 10 & 11 & 11 & 14 & 15 & 17 & $\mathbf{7 8}$ \\
Mobile phones & 7 & 10 & 14 & 19 & 25 & 28 & $\mathbf{1 0 3}$ \\
Cheating/examinations & 17 & 18 & 20 & 14 & 20 & 25 & 119 \\
Pregnancy & 6 & 9 & 8 & 9 & 10 & 12 & $\mathbf{5 4}$ \\
\hline
\end{tabular}

Source: Siaya County Director of Education office, 2015.

Records at Siaya county education office show that the number of reported indiscipline cases in the sub-counties is higher than the national average level (Table 2).

Table 2: Indiscipline Level in Schools in Ugenya, Gem and Siaya Sub-Counties compared to Siaya County and National levels (2010-2015)

\begin{tabular}{|c|c|c|c|c|c|c|c|}
\hline $\begin{array}{l}\text { Forms of } \\
\text { indiscipline }\end{array}$ & $\begin{array}{l}\text { Freq } \\
(f)\end{array}$ & $\begin{array}{c}\% \\
\text { age }\end{array}$ & $\begin{array}{c}\text { Siaya } \\
\text { County (f) }\end{array}$ & $\begin{array}{c}\text { Siaya } \\
\text { County(\%) }\end{array}$ & $\begin{array}{l}\text { National } \\
\text { level (f) }\end{array}$ & $\begin{array}{l}\text { National } \\
\text { level (\%) }\end{array}$ & $\begin{array}{l}\text { Gravity of } \\
\text { the offence }\end{array}$ \\
\hline Strikes & $71 / 168$ & 42.1 & $63 / 262$ & 24.2 & $1918 / 8600$ & 22.3 & $\begin{array}{l}\text {-Loss of property } \\
\text {-injury }\end{array}$ \\
\hline Bullying & $89 / 168$ & 53.2 & $111 / 262$ & 42.4 & $3457 / 8600$ & 40.2 & $\begin{array}{l}\text {-Mental torture } \\
\text {-physical injury }\end{array}$ \\
\hline Drug abuse & $78 / 168$ & 46.4 & 100262 & 38.2 & 28648600 & 33.3 & $\begin{array}{l}\text {-Poor performance } \\
\text {-drop out }\end{array}$ \\
\hline Phones & $103 / 168$ & 61.3 & $157 / 262$ & 60.1 & $5177 / 8600$ & 60.2 & $\begin{array}{l}\text {-Confiscation } \\
\text {-truancy }\end{array}$ \\
\hline $\begin{array}{l}\text { Cheating / } \\
\text { examinations }\end{array}$ & $119 / 168$ & 70.6 & $184 / 262$ & 70.1 & 60208600 & 70.0 & $\begin{array}{l}\text {-Unrealistic results- } \\
\text { results withheld }\end{array}$ \\
\hline Pregnancy & $54 / 168$ & 32.2 & $79 / 262$ & 30.3 & 26145160 & 30.4 & $\begin{array}{l}\text {-drop out } \\
\text {-repetition }\end{array}$ \\
\hline Total & 5141008 & $51 \%$ & $694 / 1572$ & $44.1 \%$ & $22050 / 51600$ & $42.7 \%$ & \\
\hline
\end{tabular}

Source: Ministry of Education, Science and Technology, Statistics Section, 2015.

In 1948, the United Nations Convention on the Rights of the Child resolved that children should be protected from physical punishment and non-physical forms of punishment that are cruel and degrading and thus incompatible with the convention. As a signatory to the convention, the Kenyan government therefore banned mental harassment in schools as stipulated in the Education Act, 2013. Furthermore, the Wangai report (Republic of Kenya, 2001a) also recommended the use of guidance and counseling instead of mental harassment. Records at the Siaya County 
Education Office show that from 2010 to 2015, the number of reported cases of students physical abuse by teachers was higher in Ugenya, Gem and Siaya Sub- Counties, that is, one hundred and twenty six out of one hundred and sixty eight, $(75 \%)$ than the Siaya County schools with an average of one hundred and forty two out of two hundred and sixty two (54\%). These figures imply that the level of mental harassment use in schools within Ugenya, Gem and Siaya Sub-Counties is higher than the Siaya County level and the national level. This study therefore investigated the influence of mental harassment ban on student discipline in secondary schools in Ugenya, Gem and Siaya SubCounties, Kenya.

\section{Research Hypothesis}

Ho: There is no statistically significant relationship between mental harassment ban and the level of student discipline in public secondary schools.

\section{SYNTHESIS OF LITERATURE ON INFLUENCE OF MENTAL HARASSMENT BAN ON STUDENT DISCIPLINE IN SECONDARY SCHOOLS}

The Report of the Task force on student unrest and discipline in schools in Kenya recommended that school administrators should cultivate democratic and participatory environment in their schools and encourage regular meetings with students where teachers and students are encouraged to express their views, suggestions, and grievances (Republic of Kenya, 2001). This implies that the Report of the Task force on student unrest and discipline in schools (Republic of Kenya, 2001) recommended that schools should be democratized by involving students in management of the schools. Democratization of schools has led to a large decrease in student strikes and violence in schools (Omboto, 2013). Prior to this, there was a wave of strikes and violence in schools. Students responded to oppressive, autocratic leadership by violence. It is therefore evident that students have a negative attitude towards psychological torture or mental harassment. Hence any discipline management method involving mental harassment or psychological torture will be unpleasant to students resulting in indiscipline. This is because students generally have a negative attitude towards mental harassment. In fact, the wave of student violence witnessed in schools in 1990s was attributed to mental harassment (Ajowi, 2007, Simatwa, 2007 \& Omboto, 2013). Mental harassment is any unwelcome conduct that causes emotional distress, psychological trauma, embarrassment, and mental distress to the victim. Mental harassment interferes or limits students' ability to participate in or benefit from services, activities, or opportunities offered by a school. Mental harassment includes: threats, insults, rebuking, name calling, humiliation and reprimand.

Cotton (2005) investigated the methods used by teachers to manage student discipline in primary schools. He found out that standing in class, name calling, students being send out of class and insulting the wrong doers were common. This shows that mental harassment is common in primary schools. Kirui (2012) concurs by asserting that teachers often use verbal reprimand, insults and threats to manage student discipline in schools. Gikonyo (2002), Simatwa (2007) and Ajowi (2012) found the following as some of the methods used by teachers to manage discipline in schools: exclusion, standing in class, name calling, verbal warning, negative comments, and reprimand among others. Although the Children Act outlaws the use of mental harassment in schools, it does not specify what methods of discipline management qualify as mental harassment. It leaves it to the teacher to interpret and decides which methods will be regarded as mental harassment. The teachers are the implementers of policies at the school level and they can only implement the policies that they are able to interpret correctly (Kindiki, 2009).

Nduku (2004) and Ouma Ouma, Simatwa and Serem (2013) investigated alternative methods teachers used in the absence of corporal punishment. They concluded that teachers used various methods including mental harassment. These studies are in line with a study carried out by Simatwa (2007) who concluded that teachers use mental harassment to control minor offences. In a related study, Omboto (2012) found that teachers used methods that were punitive and illegal to manage student discipline. The methods used included mental harassment. Studies by Simatwa (2007) and Omboto (2012) investigated methods used by teachers to manage student discipline in schools. Studies by Nduku (2004) and Ouma, Simatwa and Serem (2013) investigated alternative methods to corporal punishment used by teachers to manage student discipline in schools.

\section{CONCEPTUAL FRAMEWORK}

The study adopted a conceptual framework based on Douglas McGregor's theory $Y$ (Owens, 1987) as shown in Figure 1. 
Independent Variables
Dependent Variable

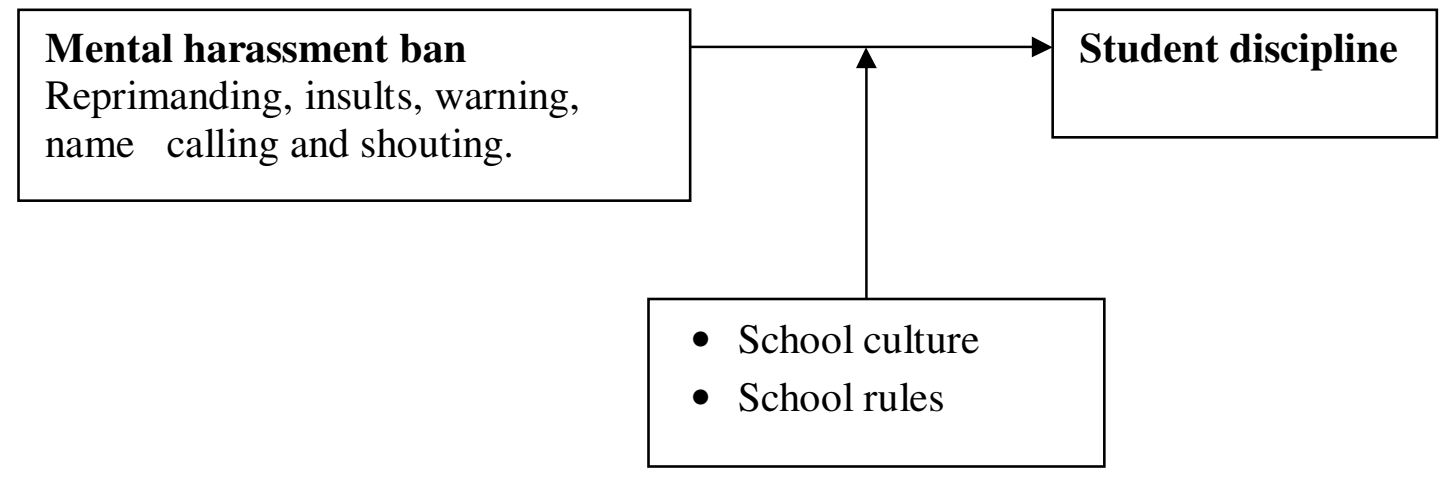

Figure 1: Conceptual Framework showing influence of mental harassment ban on student discipline

The conceptual framework (Figure 1) was based on the concept that when mental harassment is withdrawn and a conducive environment, students are disciplined. Coercion/ punishment is not needed for students to operate orderly and productively. The study investigated the influence of mental harassment ban on the level of student discipline in secondary schools. The conceptual framework postulates that mental harassment, which is the independent variable, affects the level of student discipline, which is the dependent variable. Independent variable is a variable that researchers manipulated in order to determine its effects on the dependant variable. The conceptual framework envisages that the independent variable that is mental harassment ban determines the level of student discipline in schools. From the literature reviewed, mental harassment is more effective in student discipline management compared to alternative methods like guidance and counseling. Students prefer mental harassment ban resulting in high level of discipline. The use of alternative methods of discipline management is less effective and more likely, results in high levels of offences (Busienei, 2012).

The intervening variable is one that moderates the independent variables influence on the dependent variable (Kenya Institute of Management, 2009). The conceptual framework postulates that intervening variables include school rules and school culture. For teachers to manage discipline using any discipline management method, there must be school rules in place. The school rules will guide the teachers as they manage student discipline. School culture determines which discipline management methods are acceptable in a school. Teachers' attitudes towards methods of discipline management determine whether these methods will be effective or not. Teachers are the implementers of policies at the school level (Ouma et al, 2013). Discipline management methods can only have an effect on student discipline level only if they are fully implemented. As a consequent to mental harassment ban in schools, teachers feel that they have been completely stripped of their powers and have no control over their students and they feel they have been given no alternatives. As a result they feel completely helpless (Kopansky, 2002). The attitude of teachers towards government policies on discipline affects discipline management in schools, since teachers are the implementers of policies at the school level. The government emphasizes on guidance and counseling for discipline management in schools. Teachers argue that they lack guidance and counseling skills. They therefore need in-service training in guidance and counseling for them to be able to use it effectively to manage student discipline. Teachers argue that alternative methods of discipline management like guidance and counseling take a lot of time which should be used for learning activities. They argue that such methods are only effective in schools where students have self discipline (Samoei, 2012). School culture determines whether mental harassment can be used effectively to maintain discipline in schools. In some schools, mental harassment is part of the school culture and students accept it. Mental harassment means methods that cause psychological torture like reprimanding, insulting and name calling. Head teacher's management style also determines the effective discipline management method (Kiumi, 2008). For example schools where the head teacher uses democratic style of management is likely to be inclusive, whereby, all stakeholders are involved and hence guidance and counseling method will be effective in discipline management. Suspension and expulsion are used as a last resort where other methods have failed. They are also used in cases of intolerable offences like fighting and destruction of property (Omboto, 2013). 


\section{RESEARCH METHODOLOGY}

The study was guided by Douglas McGregor's Theory Y (Owens, 1987). The study employed descriptive survey and correlational research designs. The study population was 10,629 respondents. That is, 168 principals, 168 deputy principals, 168 guidance and counseling teachers, 924 class representatives, 9200 form four students, and the Siaya County Director of Education. Stratified random sampling technique was used to select 116 principals, 116 deputy principals, 116 guidance and counseling teachers, 274 class representatives and 400 form four students. Saturation sampling was used to select the County Director of Education. The instruments of data collection were questionnaires, observation guide, interview schedule, focus group discussion guide, and document analysis guide. The content validity of the questionnaires, document analysis guide and interview schedule were addressed by research experts and their comments and suggestions were incorporated in the instruments. To enhance reliability, piloting was done in nine schools. Test-retest method was used to estimate the reliability of the instruments. Quantitative data on level of use of mental harassment ban and level of student discipline collected by use of questionnaires and document analysis guide was analyzed using frequency counts, percentages and means. Regression analysis was used to establish the influence of mental harassment ban on student discipline. Qualitative data collected by use of the in-depth interview, observation guide and focus group discussion was transcribed and arranged into themes as they emerged from the data.

\section{RESULTS}

Information on demographic characteristics of deputy principals, guidance and counseling (Guidance and Couselling) teachers and students was obtained through questionnaires and document analysis. The information was tabulated as shown in Tables 3, 4 and 5 respectively.

Table 3: Demographic Characteristics of Deputy Principals

\begin{tabular}{lcc}
\hline Demographic Data & Frequency (f) & Percentage (\%) \\
\hline Gender & 50 & 43 \\
Female & 66 & 57 \\
Male & $\mathbf{1 1 6}$ & $\mathbf{1 0 0}$ \\
Total & & \\
& & \\
Deputy headship experience & 39 & 34 \\
1-11 months & 49 & 42 \\
1-2 years & 28 & 24 \\
3-5 years & $\mathbf{1 1 6}$ & $\mathbf{1 0 0}$ \\
Total & & \\
\hline
\end{tabular}

Table 3 shows that $50(43 \%)$ deputy principals were females, while $66(57 \%)$ were males. Table 4.1 also shows that $39(34 \%)$ deputy principals had experience of $1-11$ months, $49(42 \%)$ had experience of $1-2$ years and $28(24 \%)$ had experience of 3-5 years as deputy principals. Table 4 shows $G \& C$ teachers demographic characteristics.

Table 4: Demographic Characteristics of Guidance and Counseling Teachers'

\begin{tabular}{lcc}
\hline $\begin{array}{l}\text { Demographic } \\
\text { characteristics }\end{array}$ & Frequency (f) & Percentage (\%) \\
\hline Gender & 56 & 48 \\
Female & 60 & 52 \\
Male & $\mathbf{1 1 6}$ & $\mathbf{1 0 0}$ \\
Total & & \\
G\&C Experience & 50 & 43 \\
$1-11$ months & 38 & 33 \\
$1-2$ years & 28 & 24 \\
$3-5$ years & $\mathbf{1 1 6}$ & $\mathbf{1 0 0}$ \\
Total & & \\
\hline
\end{tabular}


Fifty six (48\%) Guidance and Couselling teachers were females, while 60(52\%) were males. Table 4.2 also shows that $50(43 \%)$ Guidance and Couselling) teachers had experience of 1-11 months as Guidance and Couselling teachers and $38(33 \%)$ had experience of $1-2$ years, while $28(24 \%)$ had experience of 3-5 years. Table 5 shows class representatives demographic characteristics.

Table 5: Demographic Characteristics of Class Representatives

\begin{tabular}{lll}
\hline Demographic Data & Frequency (f) & Percentage (\%) \\
\hline Gender & & \\
Male & 152 & 56 \\
Female & 120 & 44 \\
Total Performance & $\mathbf{2 7 2}$ & $\mathbf{1 0 0}$ \\
& & \\
Above Average & 139 & 51 \\
Below Average & 133 & 49 \\
Total & $\mathbf{2 7 2}$ & $\mathbf{1 0 0}$ \\
\hline
\end{tabular}

Table 5 shows that 139 (51\%) students were above average, while $133(49 \%)$ were below average in academic performance. One hundred and fifty two (56\%) students were males and $120(44 \%)$ were females.

Table 6: Schools Data

\begin{tabular}{lcc}
\hline Category of school & Freq(f) & $\%$ \\
\hline Single-stream & 51 & 44 \\
Multi- stream & 65 & 56 \\
Total & $\mathbf{1 1 6}$ & $\mathbf{1 0 0}$ \\
\hline
\end{tabular}

Large student populations are more difficult to control than smaller populations. The schools were therefore categorized according to size. Table 6 shows that 51 (44\%) schools were single stream schools and 65 (56\%) schools were multi-stream schools.

\section{Research Hypothesis}

The hypothesis that was used to establish the influence of mental harassment ban on student discipline was: "There is no statistically significant relationship between mental harassment ban and students discipline in public secondary schools." The first step in data analysis involved descriptive analysis of the stress levels among students and the level of mental harassment ban in the three sub counties. Thus, to establish the influence of mental harassment ban on student discipline, the students' discipline level was first established. The results were as shown Table 7. 
Table 7: Level of Student Discipline as Rated by Deputy Principals, Guidance and Counseling Teachers and Students

\begin{tabular}{|c|c|c|c|c|c|c|c|c|c|c|}
\hline \multirow{2}{*}{$\begin{array}{l}\text { Indicators } \\
\text { of discipline }\end{array}$} & \multirow[t]{2}{*}{ Resp } & \multicolumn{5}{|c|}{ Level of Discipline } & \multirow[t]{2}{*}{ MR } & \multirow[t]{2}{*}{ SD } & \multirow[t]{2}{*}{ OMR } & \multirow[t]{2}{*}{ ANOVA } \\
\hline & & VH & $\mathrm{H}$ & M & $\mathbf{L}$ & VL & & & & \\
\hline \multirow[t]{3}{*}{ Vandalism } & $\mathrm{D} / \mathrm{P}$ & 4 & 21 & 21 & 44 & 26 & 2.42 & 1.128 & 2.38 & $(F(2,501)=0.365, p=0.694)$ \\
\hline & $\mathrm{G} / \mathrm{C}$ & 3 & 21 & 21 & 44 & 27 & 2.39 & 1.109 & & \\
\hline & $S$ & 8 & 44 & 44 & 108 & 68 & 2.32 & 1.106 & & \\
\hline \multirow[t]{3}{*}{ Noise making } & $D / P$ & 2 & 11 & 18 & 53 & 32 & 2.12 & 0.979 & 2.14 & $(F(2,501)=0.056, p=0.945)$ \\
\hline & $\mathrm{G} / \mathrm{C}$ & 3 & 12 & 19 & 49 & 33 & 2.16 & 1.038 & & \\
\hline & $S$ & 5 & 27 & 44 & 120 & 76 & 2.14 & 0.994 & & \\
\hline \multirow[t]{3}{*}{ Lateness } & $\mathrm{D} / \mathrm{P}$ & 10 & 20 & 22 & 40 & 21 & 2.59 & 1.238 & 2.56 & $(\mathrm{~F}(2,501)=0.070, \mathrm{p}=0.933)$ \\
\hline & $\mathrm{G} / \mathrm{C}$ & 13 & 12 & 20 & 49 & 25 & 2.53 & 1.233 & & \\
\hline & S & 27 & 38 & 49 & 104 & 54 & 2.56 & 1.235 & & \\
\hline \multirow{3}{*}{$\begin{array}{l}\text { Not doing } \\
\text { homework }\end{array}$} & $D / P$ & 6 & 20 & 23 & 45 & 22 & 2.51 & 1.138 & 2.51 & $(F(2,501)=0.333, p=0.717)$ \\
\hline & $\mathrm{G} / \mathrm{C}$ & 6 & 22 & 23 & 45 & 20 & 2.56 & 1.137 & & \\
\hline & $S$ & 14 & 44 & 49 & 111 & 54 & 2.46 & 1.132 & & \\
\hline \multirow{3}{*}{$\begin{array}{l}\text { Sleeping in } \\
\text { Class }\end{array}$} & $\mathrm{D} / \mathrm{P}$ & 3 & 21 & 20 & 35 & 37 & 2.29 & 1.172 & 2.32 & $(\mathrm{~F}(2,501)=0.117, \mathrm{p}=0.890)$ \\
\hline & $\mathrm{G} / \mathrm{C}$ & 2 & 21 & 26 & 35 & 32 & 2.36 & 1.122 & & \\
\hline & $S$ & 5 & 49 & 54 & 82 & 82 & 2.33 & 1.137 & & \\
\hline Not putting on & $\mathrm{D} / \mathrm{P}$ & 5 & 13 & 24 & 38 & 35 & 2.25 & 1.141 & 2.36 & $(F(2,501)=4.069, p=0.018)$ \\
\hline School & $\mathrm{G} / \mathrm{C}$ & 4 & 24 & 13 & 36 & 40 & 2.30 & 1.230 & & \\
\hline uniform & S & 11 & 44 & 44 & 87 & 86 & 2.29 & 0.971 & & \\
\hline Vulgar & $\mathrm{D} / \mathrm{P}$ & 13 & 20 & 20 & 41 & 22 & 2.66 & 1.278 & 2.57 & $(F(2,501)=0.524, p=0.593)$ \\
\hline \multirow[t]{2}{*}{ Language } & $\mathrm{G} / \mathrm{C}$ & 10 & 15 & 26 & 41 & 24 & 2.53 & 1.205 & & \\
\hline & $S$ & 27 & 38 & 49 & 95 & 63 & 2.53 & 1.262 & & \\
\hline \multirow{3}{*}{$\begin{array}{l}\text { Vernacular } \\
\text { speaking }\end{array}$} & $\mathrm{D} / \mathrm{P}$ & 14 & 22 & 25 & 30 & 25 & 2.74 & 1.320 & 2.70 & $(F(2,501)=0.591, p=0.554)$ \\
\hline & $\mathrm{G} / \mathrm{C}$ & 14 & 20 & 26 & 35 & 21 & 2.75 & 1.278 & & \\
\hline & S & 27 & 44 & 60 & 81 & 60 & 2.62 & 1.265 & & \\
\hline \multirow{3}{*}{$\begin{array}{l}\text { Deviant } \\
\text { behaviour }\end{array}$} & $\mathrm{D} / \mathrm{P}$ & 5 & 20 & 24 & 41 & 25 & 2.46 & 1.145 & 2.43 & $(F(2,501)=0.099, p=0.905)$ \\
\hline & $\mathrm{G} / \mathrm{C}$ & 4 & 22 & 22 & 41 & 26 & 2.44 & 1.144 & & \\
\hline & $S$ & 11 & 44 & 54 & 98 & 65 & 2.40 & 1.136 & & \\
\hline \multirow[t]{3}{*}{ Sneaking } & $\mathrm{D} / \mathrm{P}$ & 4 & 20 & 12 & 52 & 28 & 2.32 & 1.124 & 2.26 & $(\mathrm{~F}(2,501)=0.368, \mathrm{p}=0.692)$ \\
\hline & $\mathrm{G} / \mathrm{C}$ & 3 & 13 & 20 & 48 & 32 & 2.22 & 1.149 & & \\
\hline & S & 8 & 38 & 38 & 117 & 71 & 2.25 & 1.081 & & \\
\hline \multirow{3}{*}{$\begin{array}{l}\text { Boy-girl canal } \\
\text { Knowledge }\end{array}$} & $\mathrm{D} / \mathrm{P}$ & 6 & 21 & 23 & 45 & 21 & 2.53 & 1.138 & 2.56 & $(F(2,501)=0.562, p=0.571)$ \\
\hline & $\mathrm{G} / \mathrm{C}$ & 6 & 21 & 23 & 45 & 21 & 2.53 & 1.138 & & \\
\hline & $S$ & 16 & 54 & 68 & 85 & 49 & 2.51 & 1.160 & & \\
\hline \multirow{3}{*}{$\begin{array}{l}\text { Fighting in } \\
\text { school }\end{array}$} & $D / P$ & 6 & 30 & 34 & 26 & 20 & 2.79 & 1.161 & 2.85 & $(F(2,501)=0.320, p=0.726)$ \\
\hline & $\mathrm{G} / \mathrm{C}$ & 6 & 35 & 35 & 23 & 17 & 2.91 & 1.139 & & \\
\hline & S & 14 & 76 & 81 & 57 & 44 & 2.85 & 1.151 & & \\
\hline
\end{tabular}




\begin{tabular}{|c|c|c|c|c|c|c|c|c|c|c|}
\hline \multirow{2}{*}{$\begin{array}{l}\text { Indicators } \\
\text { of discipline }\end{array}$} & \multirow[t]{2}{*}{ Resp } & \multicolumn{5}{|c|}{ Level of Discipline } & \multirow[t]{2}{*}{ MR } & \multirow[t]{2}{*}{ SD } & \multirow[t]{2}{*}{ OMR } & \multirow[t]{2}{*}{ ANOVA } \\
\hline & & VH & $\mathbf{H}$ & M & $\mathbf{L}$ & VL & & & & \\
\hline \multirow[t]{3}{*}{ Delinquency } & $\mathrm{D} / \mathrm{P}$ & 7 & 10 & 21 & 56 & 22 & 2.34 & 1.072 & 2.37 & $(\mathrm{~F}(2,501)=0.120, \mathrm{p}=0.887)$ \\
\hline & $\mathrm{G} / \mathrm{C}$ & 7 & 13 & 21 & 55 & 20 & 2.41 & 1.088 & & \\
\hline & $S$ & 16 & 27 & 49 & 131 & 49 & 2.20 & 1.073 & & \\
\hline \multirow{3}{*}{$\begin{array}{l}\text { Cheating in } \\
\text { examinations }\end{array}$} & $\mathrm{D} / \mathrm{P}$ & 3 & 12 & 15 & 44 & 42 & 2.05 & 1.070 & 2.03 & $(F(2,501)=0.064, p=0.938)$ \\
\hline & $\mathrm{G} / \mathrm{C}$ & 2 & 11 & 17 & 42 & 44 & 2.02 & 1.034 & & \\
\hline & $\mathrm{S}$ & 5 & 27 & 33 & 109 & 98 & 2.02 & 1.024 & & \\
\hline \multirow[t]{3}{*}{ Drug abuse } & $\mathrm{D} / \mathrm{P}$ & 5 & 13 & 40 & 28 & 30 & 2.44 & 1.121 & 2.41 & $(F(2,501)=0.660, p=0.517)$ \\
\hline & $\mathrm{G} / \mathrm{C}$ & 4 & 15 & 39 & 30 & 28 & 2.46 & 1.099 & & \\
\hline & $\mathrm{S}$ & 8 & 27 & 82 & 87 & 68 & 2.34 & 1.050 & & \\
\hline Bullying & $\mathrm{D} / \mathrm{P}$ & 4 & 12 & 45 & 37 & 18 & 2.54 & 0.990 & 2.54 & $(F(2,501)=0.002, p=0.998)$ \\
\hline \multirow[t]{2}{*}{ School mates } & $\mathrm{G} / \mathrm{C}$ & 5 & 11 & 44 & 37 & 19 & 2.53 & 1.017 & & \\
\hline & $S$ & 11 & 27 & 103 & 87 & 44 & 2.54 & 1.009 & & \\
\hline \multirow[t]{3}{*}{ Theft in school } & $\mathrm{D} / \mathrm{P}$ & 3 & 10 & 50 & 30 & 23 & 2.48 & 0.991 & 2.48 & $(F(2,501)=1.019, p=0.362)$ \\
\hline & $\mathrm{G} / \mathrm{C}$ & 2 & 9 & 47 & 35 & 23 & 2.41 & 0.952 & & \\
\hline & $\mathrm{S}$ & 5 & 27 & 121 & 81 & 38 & 2.42 & 0.915 & & \\
\hline \multirow{3}{*}{$\begin{array}{l}\text { Disobedience } \\
\text { to teachers }\end{array}$} & $\mathrm{D} / \mathrm{P}$ & 3 & 4 & 35 & 55 & 19 & 2.28 & 0.873 & 2.29 & $(F(2,501)=0.014, p=0.986)$ \\
\hline & $\mathrm{G} / \mathrm{C}$ & 2 & 5 & 35 & 56 & 18 & 2.28 & 0.842 & & \\
\hline & $\mathrm{S}$ & 5 & 16 & 88 & 109 & 54 & 2.30 & 0.915 & & \\
\hline \multirow[t]{3}{*}{ Truancy } & $\mathrm{D} / \mathrm{P}$ & 2 & 5 & 23 & 55 & 31 & 2.07 & 0.892 & 2.10 & $(F(2,501)=0.213, p=0.808)$ \\
\hline & $\mathrm{G} / \mathrm{C}$ & 3 & 4 & 23 & 57 & 29 & 2.09 & 0.904 & & \\
\hline & S & 5 & 16 & 60 & 120 & 71 & 2.14 & 0.932 & & \\
\hline \multirow{3}{*}{$\begin{array}{l}\text { Defiance (co- } \\
\text { Curricular } \\
\text { activities) }\end{array}$} & $\mathrm{D} / \mathrm{P}$ & 3 & 11 & 20 & 55 & 27 & 2.21 & 0.991 & 2.25 & $(F(2,501)=0.475, p=0.622)$ \\
\hline & $\mathrm{G} / \mathrm{C}$ & 2 & 12 & 22 & 56 & 24 & 2.24 & 0.957 & & \\
\hline & S & 8 & 33 & 54 & 117 & 60 & 2.31 & 0.038 & & \\
\hline \multirow[t]{3}{*}{ Overall } & $\mathrm{D} / \mathrm{P}$ & 5 & 16 & 26 & 43 & 26 & 2.41 & 1.128 & 2.41 & $(F(2,501)=0.003, p=0.997)$ \\
\hline & $\mathrm{G} / \mathrm{C}$ & 5 & 16 & 26 & 43 & 26 & 2.41 & 1.128 & & \\
\hline & S & 12 & 37 & 61 & 99 & 63 & 2.40 & 1.115 & & \\
\hline
\end{tabular}

Key: $\mathrm{VH}=$ very high, $\mathrm{H}=$ high, $\mathrm{M}=$ moderate, $\mathrm{L}=\mathrm{low}, \mathrm{VL}=$ very low. Resp =respondents, $\mathrm{MR}=$ mean rate, $\mathrm{OMR}=$ overall mean rate, $\mathrm{SD}=$ standard deviation. The five point scale used was as illustrated:

\section{Interpretation of mean rating}

$\begin{array}{llc}\text { Mean ratings } & \text { Descriptor } & \text { Percentage } \\ 1.00-1.44 & \text { Very low } & 20 \\ 1.45-2.44 & \text { low } & 40 \\ 2.45-3.44 & \text { Moderate } & 60 \\ 3.45-4.44 & \text { High } & 80 \\ 4.45-5.00 & \text { Very high } & 100\end{array}$

From Table 7, it can be observed that the students' level of discipline was mean rated at 2.41. This means that the student discipline level was low at about $40 \%$. The extent to which mental harassment ban was implemented in Ugenya, Gem and Siaya sub counties was established. The results were as shown in Table 8. 
Table 8: Extent of implementation of Mental Harassment ban

\begin{tabular}{|c|c|c|c|c|c|c|c|c|c|c|}
\hline \multirow[t]{2}{*}{ Offences } & \multirow[t]{2}{*}{ Res } & \multicolumn{5}{|c|}{ MH Ban } & \multirow[t]{2}{*}{ MR } & \multirow[t]{2}{*}{ SD } & \multirow[t]{2}{*}{ OMR } & \multirow[t]{2}{*}{ ANOVA } \\
\hline & & VH & $\mathbf{H}$ & $\mathbf{M}$ & $\mathbf{L}$ & VL & & & & \\
\hline \multirow{3}{*}{ Truancy } & $\mathrm{D} / \mathrm{P}$ & 19 & 35 & 9 & 40 & 13 & 3.06 & 1.327 & 3.01 & $(F(2,501)=0.33, p=0.719)$ \\
\hline & $G / C$ & 18 & 35 & 9 & 41 & 13 & 3.03 & 1.318 & & \\
\hline & S & 41 & 76 & 22 & 95 & 38 & 2.95 & 1.340 & & \\
\hline \multirow[t]{3}{*}{ Noise } & $D / P$ & 11 & 12 & 35 & 41 & 17 & 2.65 & 1.144 & 2.64 & $(F(2,501)=0.074, p=0.929)$ \\
\hline & $\mathrm{G} / \mathrm{C}$ & 12 & 11 & 35 & 40 & 18 & 2.65 & 1.167 & & \\
\hline & S & 27 & 27 & 76 & 96 & 46 & 2.61 & 1.173 & & \\
\hline \multirow[t]{3}{*}{ Lateness } & $D / P$ & 10 & 21 & 23 & 44 & 18 & .66 & 1.94 & 2.63 & $(F(2,501)=0.107, p=0.898)$ \\
\hline & $G / C$ & 9 & 21 & 23 & 44 & 19 & 2.63 & 1.183 & & \\
\hline & $\mathrm{S}$ & 22 & 49 & 49 & 103 & 49 & 2.60 & 1.204 & & \\
\hline \multirow[t]{3}{*}{ Not homework } & $D / P$ & 18 & 37 & 21 & 29 & 11 & 3.19 & 1.244 & 3.13 & $(F(2,501)=0.688, p=0.503)$ \\
\hline & $\mathrm{G} / \mathrm{C}$ & 17 & 37 & 21 & 29 & 12 & 3.16 & 1.248 & & \\
\hline & S & 41 & 65 & 49 & 68 & 49 & 3.04 & 1.306 & & \\
\hline \multirow[t]{2}{*}{ Sleeping in class } & $D / P$ & 12 & 23 & 25 & 33 & 23 & 2.72 & 1.276 & 2.71 & $(F(2,501)=0.119, p=0.888)$ \\
\hline & $\mathrm{G} / \mathrm{C}$ & 12 & 23 & 26 & 32 & 23 & 2.73 & 1.274 & & \\
\hline \multirow{4}{*}{ Improper dressing } & S & 27 & 54 & 54 & 77 & 60 & 2.67 & 1.288 & & \\
\hline & $D / P$ & 18 & 21 & 45 & 17 & 15 & 3.09 & 1.213 & 3.05 & $(F(2,501)=0.426, p=0.653)$ \\
\hline & $\mathrm{G} / \mathrm{C}$ & 18 & 20 & 45 & 18 & 15 & 3.07 & 1.214 & & \\
\hline & S & 38 & 43 & 107 & 43 & 41 & 2.98 & 1.218 & & \\
\hline \multirow[t]{2}{*}{ Vulgar Language } & $D / P$ & 10 & 18 & 35 & 36 & 17 & 2.72 & 1.154 & 2.69 & $(F(2,501)=0.080, p=0.923)$ \\
\hline & $\mathrm{G} / \mathrm{C}$ & 9 & 17 & 35 & 37 & 18 & 2.67 & 1.140 & & \\
\hline \multirow{4}{*}{ Mother tongue } & $\mathrm{S}$ & 22 & 41 & 82 & 81 & 46 & 2.68 & 1.161 & & \\
\hline & $D / P$ & 10 & 16 & 32 & 35 & 23 & 2.61 & 1.200 & 2.59 & $(F(2,501)=0.028, p=0.972)$ \\
\hline & $\mathrm{G} / \mathrm{C}$ & 9 & 16 & 33 & 35 & 23 & 2.59 & 1.179 & & \\
\hline & S & 22 & 38 & 76 & 76 & 60 & 2.58 & 1.206 & & \\
\hline \multirow[t]{3}{*}{ Disruptive } & $\mathrm{D} / \mathrm{P}$ & 11 & 18 & 23 & 35 & 29 & 2.54 & 1.281 & 2.54 & $(F(2,501)=0.013, p=0.987)$ \\
\hline & $\mathrm{G} / \mathrm{C}$ & 12 & 17 & 23 & 35 & 29 & 2.55 & 1.294 & & \\
\hline & $\mathrm{S}$ & 27 & 41 & 54 & 77 & 73 & 2.53 & 1.300 & & \\
\hline \multirow[t]{3}{*}{ Sneaking } & $\mathrm{D} / \mathrm{P}$ & 23 & 32 & 37 & 14 & 10 & 3.38 & 1.184 & 3.38 & $(F(2,501)=0.069, p=0.933)$ \\
\hline & $\mathrm{G} / \mathrm{C}$ & 23 & 33 & 37 & 14 & 9 & 3.41 & 1.165 & & \\
\hline & S & 54 & 76 & 82 & 33 & 27 & 3.36 & 1.212 & & \\
\hline \multirow[t]{3}{*}{$\mathrm{B} / \mathrm{G}$ relationship } & $D / P$ & 18 & 32 & 35 & 14 & 17 & 3.17 & 1.260 & 3.16 & $(F(2,501)=0.012, p=0.988)$ \\
\hline & $\mathrm{G} / \mathrm{C}$ & 17 & 33 & 34 & 14 & 18 & 3.15 & 1.267 & & \\
\hline & S & 41 & 76 & 81 & 33 & 41 & 3.16 & 1.260 & & \\
\hline \multirow[t]{3}{*}{ Disobedience } & $\mathrm{D} / \mathrm{P}$ & 6 & 12 & 25 & 41 & 32 & 2.30 & 1.136 & 2.29 & $(F(2,501)=0.007, p=0.993)$ \\
\hline & $\mathrm{G} / \mathrm{C}$ & 6 & 11 & 26 & 40 & 33 & 2.28 & 1.133 & & \\
\hline & S & 16 & 27 & 54 & 99 & 76 & 2.29 & 1.150 & & \\
\hline \multirow[t]{3}{*}{ Delinquency } & $D / P$ & 6 & 11 & 35 & 35 & 29 & 2.40 & 1.118 & 2.40 & $(\mathrm{~F}(2,501)=0.002, \mathrm{p}=0.998)$ \\
\hline & $\mathrm{G} / \mathrm{C}$ & 6 & 12 & 34 & 35 & 29 & 2.41 & 1.127 & & \\
\hline & S & 14 & 27 & 82 & 81 & 68 & 2.40 & 1.119 & & \\
\hline
\end{tabular}




\begin{tabular}{|c|c|c|c|c|c|c|c|c|c|c|}
\hline \multirow[t]{2}{*}{ Offences } & \multirow[t]{2}{*}{ Res } & \multicolumn{5}{|c|}{ MH Ban } & \multirow[t]{2}{*}{ MR } & \multirow[t]{2}{*}{ SD } & \multirow[t]{2}{*}{ OMR } & \multirow[t]{2}{*}{ ANOVA } \\
\hline & & VH & $\mathbf{H}$ & M & $\mathbf{L}$ & $\overline{\mathbf{V L}}$ & & & & \\
\hline \multirow[t]{3}{*}{ Cheating } & $\mathrm{D} / \mathrm{P}$ & 11 & 33 & 49 & 11 & 12 & 3.17 & 1.074 & 3.16 & $(F(2,501)=0.251, p=0.778)$ \\
\hline & $\mathrm{G} / \mathrm{C}$ & 12 & 32 & 49 & 12 & 11 & 3.19 & 1.071 & & \\
\hline & S & 24 & 73 & 115 & 30 & 30 & 3.11 & 1.079 & & \\
\hline \multirow[t]{3}{*}{ Drug abuse } & $D / P$ & 23 & 30 & 42 & 11 & 10 & 3.39 & 1.163 & 3.35 & $(F(2,501)=0.694, p=0.500)$ \\
\hline & $\mathrm{G} / \mathrm{C}$ & 23 & 30 & 42 & 12 & 9 & 3.40 & 1.149 & & \\
\hline & S & 54 & 54 & 99 & 41 & 24 & 3.27 & 1.196 & & \\
\hline \multirow[t]{3}{*}{ Bullying } & $D / P$ & 6 & 12 & 31 & 35 & 32 & 2.35 & 1.144 & 2.33 & $(F(2,501)=0.082, p=0.922)$ \\
\hline & $\mathrm{G} / \mathrm{C}$ & 6 & 11 & 32 & 34 & 33 & 2.34 & 1.142 & & \\
\hline & S & 14 & 27 & 68 & 82 & 81 & 2.31 & 1.149 & & \\
\hline \multirow[t]{3}{*}{ Theft } & $D / P$ & 14 & 22 & 49 & 19 & 12 & 3.06 & 1.121 & 3.13 & $(F(2,501)=0.924, p=0.147)$ \\
\hline & $\mathrm{G} / \mathrm{C}$ & 14 & 22 & 49 & 20 & 11 & 3.07 & 1.109 & & \\
\hline & S & 46 & 52 & 114 & 46 & 14 & 3.26 & 1.087 & & \\
\hline \multirow[t]{3}{*}{ Fighting/violence } & $D / P$ & 14 & 22 & 51 & 18 & 11 & 2.35 & 1.121 & 2.33 & $(F(2,501)=0.293, p=0.746)$ \\
\hline & $\mathrm{G} / \mathrm{C}$ & 14 & 22 & 51 & 17 & 12 & 2.34 & 1.109 & & \\
\hline & $S$ & 33 & 52 & 108 & 41 & 38 & 2.31 & 1.087 & & \\
\hline \multirow[t]{3}{*}{ Vandalism } & $\mathrm{D} / \mathrm{P}$ & 6 & 11 & 35 & 35 & 29 & 2.40 & 1.118 & 2.39 & $(F(2,501)=0.033, p=0.968)$ \\
\hline & $\mathrm{G} / \mathrm{C}$ & 6 & 12 & 35 & 34 & 29 & 2.41 & 1.127 & & \\
\hline & $S$ & 14 & 27 & 76 & 87 & 68 & 2.38 & 1.117 & & \\
\hline \multirow[t]{3}{*}{ Defiance } & $\mathrm{D} / \mathrm{P}$ & 11 & 18 & 26 & 37 & 24 & 2.61 & 1.243 & 2.57 & $(F(2,501)=0.924, p=0.398)$ \\
\hline & $\mathrm{G} / \mathrm{C}$ & 12 & 17 & 27 & 37 & 23 & 2.64 & 1.247 & & \\
\hline & S & 27 & 41 & 41 & 87 & 76 & 2.47 & 1.308 & & \\
\hline \multirow[t]{3}{*}{ Overall } & $D / P$ & 13 & 22 & 33 & 29 & 19 & 2.84 & 1.237 & 2.81 & $(F(2,501)=0.104, p=0.901)$ \\
\hline & $\mathrm{G} / \mathrm{C}$ & 13 & 21 & 33 & 29 & 20 & 2.82 & 1.234 & & \\
\hline & $S$ & 30 & 48 & 75 & 69 & 50 & 2.78 & 1.250 & & \\
\hline
\end{tabular}

Key: $\mathrm{VH}=$ =ery high, $\mathrm{H}=$ high, $\mathrm{M}=$ moderate, $\mathrm{L}=$ low, $\mathrm{VL}=$ =rery low, $\mathrm{MR}=$ mean rate.

$\mathbf{R e s p}=$ respondent, $\mathbf{O M R}=$ overall mean rate, $\mathbf{S D}=$ standard deviation, $\mathbf{S}=$ students,

D/P=Deputy Principals, G/C-=Guidance \& Counseling teachers.

The five point scale used was as illustrated:

\section{Interpretation of mean rating}

$\begin{array}{llc}\text { Mean ratings } & \text { Descriptor } & \text { Percentage } \\ 1.00-1.44 & \text { Very low } & 20 \\ 1.45-2.44 & \text { low } & 40 \\ 2.45-3.44 & \text { Moderate } & 60 \\ 3.45-4.44 & \text { High } & 80 \\ 4.45-5.00 & \text { Very high } & 100\end{array}$

From Table 8, it can be observed that the level of mental harassment ban in Ugenya, Gem and Siaya Sub-counties was mean rated at 2.81 . This means that the level of mental harassment ban was at about $60 \%$ student discipline level was low at about $40 \%$. The data on the level of mental harassment ban and student level of discipline ban was correlated using Pearson Product Moment Correlation coefficient to determine the strength and direction of relationship so as to infer the influence of mental harassment ban on student discipline. The results were as shown in Table 9.

Pearson's $r$ correlation coefficient was computed to establish the relationship between mental harassment ban and students discipline. The results were as shown in Table 10. 
Figure 10: Correlation Analysis of the Influence of Mental Harassment Ban and the Level of Student Discipline

\begin{tabular}{llc}
\hline & & Student discipline \\
\hline Mental harassment ban & Pearson Correlation & .828 \\
& Sig. (2-tailed) & .000 \\
& $\mathrm{~N}$ & 504 \\
\hline
\end{tabular}

From Table 10 it can be noted that there was a strong, positive and significant relationship between mental harassment ban and student discipline $(r=.828, N=504, p<.05)$.

To estimate the influence of mental harassment ban on student discipline, coefficient of determination was computed. The results were as shown in Table 11.

Table 11: Regression analysis of the influence of Mental Harassment Ban and the Level of Student Discipline

\begin{tabular}{ccccc}
\hline Model & $\mathbf{R}$ & R Square & $\begin{array}{c}\text { Adjusted } \\
\text { R Square }\end{array}$ & $\begin{array}{c}\text { Std. } \\
\text { Error of the Estimate }\end{array}$ \\
\hline 1 & $.828^{\mathrm{a}}$ & .685 & .685 & .625 \\
& & & & \\
\hline
\end{tabular}

a. Predictors: (Constant), extent of mental harassment ban implementation

From Table 11, it can be observed that mental harassment ban accounted for $68.5 \%$ of variation in student disciple line as signified by the Adjusted $R$ square $\left(R^{2}\right) .685$. This implied that $68.5 \%$ of the total variance in the level of student discipline was accounted for by mental harassment ban. The other $31.5 \%$ was due to other factors.

To establish whether mental harassment ban was a predictor of student discipline, ANOVA was computed. The results were as shown in Table 12.

Table 12: ANOVA of Mental Harassment Ban and Student Discipline

\begin{tabular}{llccccc}
\hline Model & & $\begin{array}{c}\text { Sum of } \\
\text { Squares }\end{array}$ & df & Mean Square & F & Sig. \\
\hline 1 & Regression & 427.223 & 1 & 427.223 & $1.093 \mathrm{E} 3$ & $.000^{\mathrm{a}}$ \\
& Residual & 196.205 & 502 & .391 & & \\
& \multicolumn{1}{l}{ Total } & 623.429 & 503 & & & \\
\hline a. Predictors: (Constant), & mental harassment ban & & & \\
b. Dependent Variable: & Student discipline
\end{tabular}

From Table 12, it can be noted that mental harassment ban was a predictor of student discipline $(F(1,502) 1.093$, $\mathrm{p}<.05)$.

To establish the actual influence mental harassment ban on student discipline, simple regression was computed. The results were as shown in Table 13. 
Table 13: Simple Regression analysis of mental harassment Ban and the Level of Student Discipline

\begin{tabular}{|c|c|c|c|c|c|c|}
\hline \multirow{2}{*}{\multicolumn{2}{|c|}{ Model }} & \multicolumn{2}{|c|}{$\begin{array}{l}\text { Unstandardized } \\
\text { Coefficients }\end{array}$} & \multirow{2}{*}{$\begin{array}{c}\text { Standardized } \\
\text { Coefficients } \\
\text { Beta }\end{array}$} & \multirow[b]{2}{*}{$\mathbf{t}$} & \multirow[b]{2}{*}{ Sig. } \\
\hline & & B & Std. Error & & & \\
\hline \multirow[t]{2}{*}{1} & (Constant) & .331 & .069 & & 4.819 & .000 \\
\hline & $\begin{array}{l}\text { extent of mental } \\
\text { harassment ban }\end{array}$ & .741 & .022 & .828 & 33.062 & .000 \\
\hline
\end{tabular}

Dependent Variable: level of student discipline

Pearson correlation between the variables; mental harassment ban and the level of student discipline, is 0.828 .

From Table 13, it can be noted that one unit increase in mental harassment ban can improve students discipline by .331 units. The regression equation is $\mathrm{Y}=.331+.741 \mathrm{X}$

A scatter plot was used to illustrate the relationship between mental harassment ban and student discipline. (Figure 2)

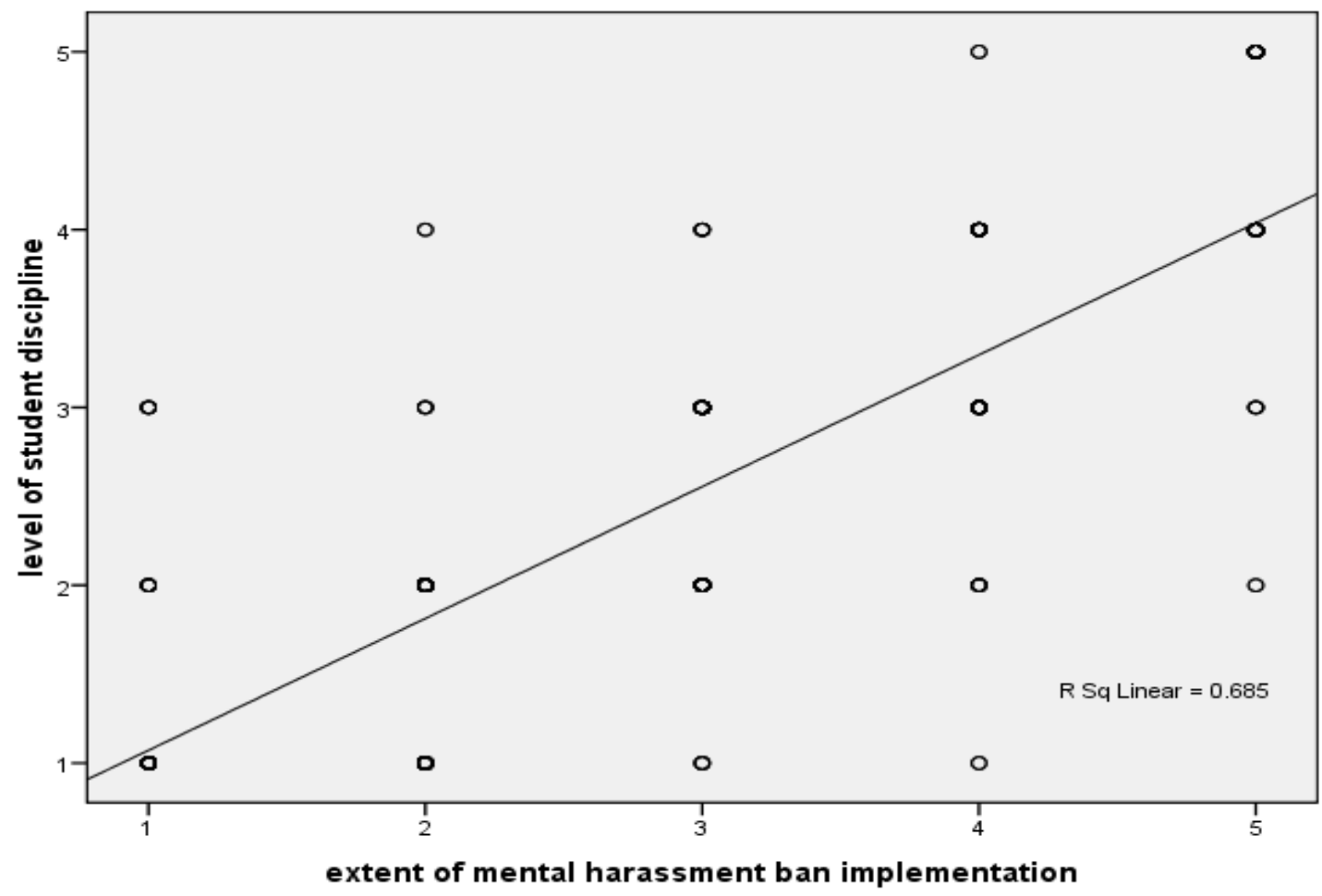

Figure 2: Scatter plot illustrating the relationship between mental harassment ban and student discipline

The scatter plot indicates that there was a linear positive relationship between mental harassment ban and student discipline. That is, increase in implementation of mental harassment ban increases the level of student discipline. This means that if the ban is fully implemented the level of student discipline will be very high.

\section{DISCUSSION}

Deputy principals, guidance and counseling teachers, and class representatives rated the extent of mental harassment implementation as moderate with respect to sneaking. This indicates that mental harassment is 
moderately used to control sneaking. Sneaking is a major offence and students who sneak from school are suspended from school (Gikonyo, 2002). Deputy principals, guidance and counseling teachers and class representatives rated the extent of mental harassment implementation to be moderate, with respect to its use to control drug abuse. This indicates that mental harassment is moderately used to control drug abuse. Students who take drugs are usually sent home to call their parents (Gikonyo, 2002). Deputy principals, Guidance and Couselling teachers and class representatives rated the extent of mental harassment ban implementation at moderate with respect to its use on students who do not do homework. This indicates that mental harassment is moderately used to punish students who do not do homework. Deputy principals, Guidance and Couselling teachers and class representatives rated the extent of mental harassment ban implementation at moderate with respect to truancy. This indicates that mental harassment is moderately used to control truancy. Truancy is a minor offence in school. With mental harassment ban, teachers tend to ignore minor offences like truancy (Miriti, 2008, Mophosa \& Shumba, 2010).

Deputy principals, guidance and counselling teachers and class representatives rated the extent of mental harassment ban implementation to be low, with respect to disobedience. This indicates that mental harassment is highly used to control disobedience. The researcher witnessed several instances in different schools whereby teachers on duty gave orders as they shouted and threatened the students who disobeyed them with dire consequences. Deputy principals, guidance and counseling teachers and class representatives rated the extent of mental harassment ban implementation to be low with respect to disobedience. This indicates that mental harassment is highly used to control disobedience in schools. Equally rated low was fighting in school which was also rated at 2.33. Deputy principals, guidance and counseling teachers and class representatives rated the extent of mental harassment ban implementation to be low with respect to fighting in school. This indicates that mental harassment is highly used to control fighting in school. Deputy principals, guidance and counseling teachers and class representatives rated the extent of mental harassment ban implementation to be low, with respect to vandalism. This indicates that mental harassment is highly used to control vandalism in school. During the focus group discussion a student lamented: "Some teachers shout at students and others even abuse them when they disobey the teachers." The researcher observed many cases where teachers on duty abused students while they supervised manual work. This was common in big schools with large student population. During the focus group discussion, it came out clearly that students seemed not to be aware of mental harassment ban. The teachers could be taking advantage of this. Take for example a student who said: "I think the new constitution does not allow teachers to cane students. I do not think this includes shouting at students and warning them."

Mental harassment ban has not been fully implemented in schools. Teachers are still using mental harassment to manage discipline in schools. This finding is supported by Simatwa (2007) who found that teachers used mental harassment to control minor offences in schools. Further, evidence came from a principal who commented on mental harassment ban: "Teachers know about mental harassment ban but they do not take it seriously. A parent may take legal action against a teacher who canes his child and hurts him but not one who reprimands the child." Indeed the researcher observed several cases where teachers pointed fingers at students and in some cases calling them names and threatening them. Another principal added: "The Education Act does not clearly state which methods are categorized as mental harassment. Sometimes, teachers may avoid using the cane but they may resort to methods considered as mental harassment without actually knowing." This shows that sometimes teachers may violate mental harassment ban unknowingly. The study established that mental harassment ban in Ugenya, Gem and Siaya sub-counties was moderate. This finding concurs with studies carried out by Gikonyo (2002, Nduku (2004), Simatwa (2007) and Ouma et al (2013). These studies investigated the methods used by teachers to manage discipline in schools but they did not investigate the extent of mental harassment ban in schools. The current study established that mental harassment ban in schools in Siaya, Gem and Ugenya Sub Counties was moderate. Therefore this is the new knowledge generated by this study.

Schools that have implemented mental harassment ban have high level of discipline. In these schools, teachers use alternative methods of discipline management like guidance and counseling (Busienei, 2012). Since these methods are learner friendly, the students will cooperate with the teachers. The students will not rebel against methods that they find acceptable and pro-human rights (Republic of Kenya, 2001). Furthermore, the guidance and counseling will make students to be self disciplined (Masitsa, 2008). The related studies did not relate mental harassment ban and level of student discipline. The current study did establish that there is a high positive relationship between the level of mental harassment ban and student discipline.

The Report of the Task force on student unrest and discipline in schools in Kenya recommended that school administrators should cultivate democratic and participatory environment in their schools and encourage regular meetings with students where teachers and students are encouraged to express their views, suggestions, and grievances (Republic of Kenya, 2001). This implies that the Report of the Task force on student unrest and discipline in schools (Republic of Kenya, 2001) recommended that schools should be democratized by involving students in management of the schools. Democratization of schools has led to a large decrease in student strikes and violence 
in schools (Omboto, 2013). Prior to this, there was a wave of strikes and violence in schools. Students responded to oppressive, autocratic leadership by violence. It is therefore evident that students have a negative attitude towards psychological torture or mental harassment. Hence any discipline management method involving mental harassment or psychological torture will be unpleasant to students, resulting in indiscipline. The findings of this Tusk force support the findings of the current research in that both reveal that mental harassment ban will result in high level of student discipline. Gikonyo (2002), Simatwa (2007) and Ajowi (2012) found that the following as some of the methods used by teachers to manage discipline in schools: exclusion, standing in class, name calling, verbal warning, negative comments, and reprimand among others. Kirui (2012) concurs by asserting that teachers often use verbal reprimand, insults and threats to manage student discipline in schools to control minor offences in schools. With the enactment of mental harassment ban, teachers tend to ignore minor offences resulting in low level of student discipline (Mophosa \& Sumba, 2010). These research studies support the current research in that low extent of mental harassment ban result in low level of student discipline in schools.

During the focus group discussion, one student contributed on mental harassment by saying: "When we joined this school, teachers used to insult and abuse us a lot. We were humiliated and reprimanded on assemblies and in class rooms. Worse was for those who performed poorly in tests and examinations. They could be paraded before other students and called names. Peers could laugh at us and we got quite embarrassed. Nowadays, teachers just pass as students make noise and even some students do not do homework. I see form one, students coming to school late and the teachers on duty just look at them. In fact, that it is why there is too much noise in the lower forms." This contribution by the student shows that teachers are no longer using mental harassment to control minor offences. Instead teachers just ignore the minor offences. The students attribute this to an increase in indiscipline cases like noise making, students not doing homework and lateness. This finding tends to contradict the findings of the current research. Teachers seem to have implemented mental harassment ban but the level of discipline is low. This discrepancy can be explained by the fact that though teachers have stopped using mental harassment they just ignore minor offences. The teachers should implement mental harassment ban by taking the legal corrective measures to make students stop committing minor offences. For example, guidance and counseling will make students understand their mistakes and make effort to behave well as per the school rules and regulations. Merely ignoring minor offences is unethical and cannot make a student behave well (Dietz, 2000). The cited studies did not relate mental harassment ban and level of student discipline. The current study did establish that there was a high positive relationship between level of mental harassment ban and student discipline in Ugenya, Gem and Siaya Sub-counties.

It is important to note that as mental harassment ban increased (became more positive), the level of students discipline increased. The study found that the overall mental harassment ban rating was $2.819(60 \%)$ which corresponded to moderate ban and the level of student discipline was rated at 2.41(40\%), which corresponded to low level of discipline in Ugenya, Gem and Siaya-Sub counties. The expectation was that moderate mental harassment ban would have resulted in moderate level of student discipline. But according to the findings of the study, the level of student discipline was low. This discrepancy between the real results and the expected outcome could be explained by the fact that mental harassment ban accounted for $68.5 \%$ of students' level of discipline and other factors accounted for $31.5 \%$, hence the discrepancy.

\section{CONCLUSION}

The study established that the extent of mental harassment ban in schools in Ugenya, Gem and Siaya Counties was moderate with an overall mean rate of $2.81(60 \%)$. The study established that there was a strong relationship between mental harassment ban and the level of student discipline in secondary schools. Thus, the higher the extent of mental harassment ban, the higher the level of discipline. According to the findings of this study, the low level of student discipline in Ugenya, Gem and Siaya Sub-Counties could be attributed to a moderate level of mental harassment ban in management of student discipline. Student discipline level was low probably due to the fact that mental harassment ban had not been fully implemented.

\section{RECOMMENDATIONS}

There is need for schools to implement mental harassment ban as stipulated in The Basic Education Act, 2013. The government should ensure that this policy is implemented in all schools. This can be done by Quality Education and Standards Officers frequently visiting schools to ensure that all schools comply with the requirements of the ban. They should also organize follow up visits which would ensure that teachers fully implement mental harassment ban. 
Students should be sensitized on mental harassment ban. They should be enlightened that is, on what constitutes mental harassment ban. This would prevent teachers from taking advantage of students' ignorance and violating the ban as students would be in a position to caution them on the violation.

The government should organize in-service training courses to further train teachers on student discipline management using the alternative methods like guidance and counseling to help them steer away from use of mental harassment.

Teacher training colleges and universities should train teachers on student discipline management using alternative methods to mental harassment. The curriculum can be designed to include this aspect of management.

The government should solicit teachers' views on the problems and challenges they face in implementing mental harassment ban in school and discuss with the teachers, the solutions to these problems and the way forward.

Benchmarking trips should be organized for teachers whereby teachers would visit schools that have successfully implemented mental harassment ban. This will motivate teachers to try it in their respective schools.

\section{REFERENCES}

Aluru, L. (2013, February 2). "Four Maranda students held over school fire." Standard Digital. Nairobi: Nation Media Group. p.1.

Afullo, P.T.O. (2007). Role of Teachers in the Management of Student Discipline in Public Schools in Bondo District. Unpublished Master's thesis. Maseno University, Kenya.

Ajayi J. A. (2009). Parents Involvement in School Administration as a Correlate of Effectiveness of Secondary Schools in Nigeria. Journal of Educational Administration and Policy Studies, 18 (3), 41-46.

Ajowi, J.O. (2005). The Role of Guidance and Counseling in Promoting Student Discipline in Secondary Schools in Kisumu District. Unpublished Master's thesis. Maseno University, Kenya.

Aziza, A. (2001). Expulsion of Learners from Secondary Schools in Western Cape: Trends and Reasons ( Unpublished Master's dissertation. University of South Africa.

Blandfords, S. (2000). Managing Discipline in Schools. London: Routlegde.

Borg, W.R., Gall, P.J. \& Gall, D.M. (1993). Applying Education Research. New York: Longman.

Brister, C. (1999). Corporal Punishment in Australian Schools. Available: url:http://www.nt/gov.au.

Burgess, R.G. (2000). In the Field. An Introduction to Field Research. London: George Allan.

Busienei, A. (2012). Alternative Methods to Corporal Punishment and their Efficacy. Journal of Emerging Trends in Educational Research and Policy Studies, 3 (2), 155-161.

Butler, B. (1999). Problems of Childhood and Adolescence. London: Macmillan Press.

Cheruiyot, E. (2001). A Study of Discipline Problems Affecting Kabianga Secondary School in Belgut Division, Kericho District. Unpublished P.G.D.E. Research Project. Maseno University, Kenya.

Chumo, D. (2008). Effect of Withdrawal of Corporal Punishment on Primary School Pupils' Performance: A Case of Eldoret West District, Kenya.( Master's thesis). Mount Kenya University, Kenya.

Cicognani, L. (2004). To Punish or Discipline? Teachers Attitudes towards the Abolition of Corporal Punishment Unpublished Master's thesis. Johannesburg University, South Africa.

Cotton, K. (2005). School wide and Classroom Discipline. Portland: Northeast Educational Laboratories

Daily Post. (2013, October $16^{\text {th }}$ ). 'Ambira high school closed indefinitely'. Daily Post. p.16.

Dietz, T.L. (2000). Disciplining Children: Characteristics associated with the use of Corporal Punishment. Child Abuse and Neglect, 24(12), 1529-1542

Daily Nation. (2008, July $23^{\text {rd }}$ ). 'The government has banned the use of mobile phones in schools by students'. Daily Nation. Nairobi: Nation Media Group. p.3

Damien, M.M. (2012). Attitudes of Stakeholders towards Physical Punishment on Pupils of International and National Schools in Kampala District, Uganda. World journal of Education, 2(1), 46-62.

Duda, F.D. (1996) Guidance and Counseling in Schools: A Case Study in Machakos Municipality. Unpublished P.G.D.E. Thesis. Kenyatta University.

Elliot, D.S. (1999). The Effect of Neighbourhood Discipline on Adolescence Development. Journal of Research in Crime and Delinquency, 33(4), 389-426.

Fraenkel, R.J. \& Wallen, E.M. (2009). How to Design and Evaluate Research in Education. New York : MacGraw Hill Inc.

Gay, L. (1996). Educational Research Competence for Analysis and Application. New York: Macmillan

Gichuru, J. (2005). A Study on the Impact of the Ban on Corporal Punishment on Discipline of Students in Public Secondary Schools in Kikuyu Division, Kiambu District (Master's thesis). University of Nairobi, Kenya. 
Gikonyo, O.M. (2002). Drug abuses and Parental Knowledge of Drugs in Nairobi Province, Kenya (Master's thesis). Kenyatta University, Kenya.

Gitonga, K. (2011, September 30). 'Police sent to school after riots'. Daily Nation, p.32. Nairobi; Nation Media Group

Gladwell, A. (1999). A Survey of Teachers' Attitudes towards Corporal Punishment after the Abolishment o Corporal Punishment (Master's Dissertation). University of Western Cape, Bellville.

Griffin, G. (2005). School Mastery: Straight Talk about Boarding School Management in Kenya. Nairobi: Lectern Publications.

Grinnel, M.J. (1993). Social Work Research and Evaluation. Illinois: Peacock Publishers.

Harber, C. (2001). Schooling and Violence in South Africa: Creating a softer School. Intercultural Education, 12(1), 261-271.

Hornsby, G. (2003). Teachers and Counseling. Routledge: Falmer.

Human Rights Watch (2005). Spare the Child. Available: http://www.hrw.org

Indoshi, F.C. (2003) "Teachers experiences of the Problem period of Teaching in Kenya: Implications for teacher Induction policies and Programs." Journal of In-Service Education: An International Journal of Professional Development, 29(3), 473-488.

lqbal, N. (2003). Rights-Pakistan: Call to spare the rod in schools grows louder. New York: Global Information Network.

Kaburu, L.W. (2006). Effectiveness of Guidance and Counseling Program in Combating Indiscipline in Secondary Schools: A Case of Nakuru Municipality, Kenya (Unpublished Master's thesis). Egerton University, Kenya.

Kariuki, W. E. (2008). Factors that Lead to Secondary School Students' Unrests in Kigumo District, Murang'a County (Master's thesis). Mount Kenya University, Kenya.

Kenya Institute of Management. (2009) Fundamentals of Management Research Methods Nairobi: Macmillan

Kerlinger, F.N. (2007). Foundations of Behavioural Research. London: Holt and Winston

Kiarie, J. (2012). Indiscipline; Now Parents Perpetuate Vice. The Standard Online Available online http:// Digital std.org

Kilonzo, J. (2009). Challenges Faced by Head teachers in the Management of Students Indiscipline in Public Secondary Schools in Lamu County (Unpublished Master's thesis). Kenyatta University, Kenya.

Kindiki, J.N. (2009). Effectiveness of Communication on Students Discipline in Secondary Schools in Kenya. Educational Research and Review, 4(5): 252- 259.

Kiprop, C, J. (2004). Approaches to Management of Discipline in Secondary Schools in Kenya (Unpublished PhD thesis). Moi University, Kenya.

Kiptela, C., Okero, L. \& Kiprutu, M. (2011). Students Perceptions of Corporal Punishment in Taita Taveta County, Kenya (Unpublished Master's thesis). Egerton University, Kenya.

Kiumi, J.K. (2008). Relationship between the Principals' Management Approaches and Level of Students' Discipline in Public Secondary Schools in Nyandarua and Laikipia Districts, Kenya (Unpublished PhD thesis). Egerton University, Kenya.

Kopansky, T.M. (2002). Corporal Punishment in Schools: A hit or Miss Proposition London: Longman

Kurui, C. (2012). Influence of School Management Strategies on Students Discipline: A Case of Secondary Schools in Mogotio District, Kenya (Unpublished Master's thesis). Mount Kenya University, Kenya.

Mac Donald, M.I. (1997). Violence in Schools: Multiple Realities. Journal of Educational Research 3(2), 142-155.

Masau, K.J. (2012). Emerging Issues as a Result of Banning Corporal Punishment as a Disciplinary Method in Primary Schools in Wote Division Makueni District (Unpublished P.G.D.E. Project). Kenyatta University, Kenya.

Masha, J. (2013, August19). 'Survey reveals minors taking alcohol'. Digital Standard p.1 Available: http.//std.news.org

Masitsa, G. (2008). Discipline and disciplinary measures in the Free State Township schools: Unresolved problems. Acta Academica, 40(2), 234-270

Maxwell W.S. (1999). Teachers Attitudes towards Disruptive Behavior in Secondary Schools. Educational Review, 39(3), 203-216.

Mbindyo, M.N. (2006). Discipline Management and Control as Perceived by Students in Machakos District Kenya (Unpublished P.G.D.E. Thesis). Maseno University, Kenya.

Mbiti, M.D. (2002). Foundations of School Administration. Nairobi: Oxford University Press

Miriti, M.J. (2008). Challenges Facing School Administrators in Enforcing Students' Discipline in Public Secondary Schools in Yatta District, Machakos County (Master's thesis). Mount Kenya University, Kenya.

Mkangi, K. (2001, August 6). 'Characteristics that can lead to students' violence'. The Standard p.18. Nairobi: The Standard Group.

Mophosa, C. \& Shumba, A. (2010). Educators' Disciplinary Capabilities After the Banning of Corporal Punishment in South African Schools. South African. Journal of Education, 30(2), 387-399. 
Morrel, R. (2001). Corporal Punishment in South African Schools: A Neglected Explanation for its Persistence. South African Journal of Education, 21(3), 292-299.

Mtsweni, J. (2008). The Role of Educators in the Management of School Discipline in the Nkangala region of Mpumalanga (Master's dissertation). University of South Africa.

Mudemb, E.V. (2010). Causes of Drop out Among Boys and Girls from Secondary Schools in Ugenya District, Siaya County, Kenya (Unpublished Master's thesis). University of Nairobi, Kenya.

Mugenda, O.M. and Mugenda, A.G. (2003). Research Methods: Quantitative and Qualitative Approaches. Nairobi: Act Press

Murithi, E.W. (2010). Challenges Principals Face in Enhancing Student Discipline in Secondary Schools in Tagania District, Kenya Unpublished Master's thesis. Chuka University, Kenya.

Mutala, J.M. (2008). Challenges Faced by Secondary School Students in Learning Mathematical Concepts in Makueni County, Kenya Unpublished Master's thesis. Mount Kenya University, Kenya.

NACADA, (2004). Youth in Peril. Nairobi: Government Printers

Naong, M. (2007). The Impact of abolition of Corporal Punishment on the Teacher Morale. South African Journal of Education, 27(2), 283- 300.

Nduku, M.J. (2004). An investigation into Alternative Strategies of Discipline in the Absence of Corporal Punishment in the Public Secondary Schools in Matungulu Division, Machakos, District, Kenya Unpublished Master's thesis. University of Nairobi, Kenya.

Njambi, N. (2008). Influence of Students' Discipline on Performance in K.S.C.E. in Nakuru Municipality, Kenya. Unpublished Master's thesis. Mount Kenya University, Kenya.

Nyagah, N.E. (2008). Influence of Students Discipline on Performance in K.C.S.E. in Public Secondary Schools in Nakuru Municipality, Kenya Unpublished Master's thesis. Mount Kenya University, Kenya.

Ogemba, P. (2011, November 18). 'Students told to shun drugs'. Daily Nation, p. 9. Nairobi: Nation Media Group.

Ogetange, T.B. (2012).Teachers and Pupils' Views on Persistent use of Corporal Punishment in Managing Discipline in Primary Schools in Starehe Division, Kenya. International Journal of Humanities and Social Sciences, 19(2), 23-42.

Oketch, W. (2011, September 28). 'Students sent home at night'. The Standard, p. 28.

Ombiro, D. (2008).Challenges Facing Teachers in Enhancing Discipline among School Students in Kuria West, Migori County. Masters Thesis: Mount Kenya University.

Omboto, J.C. (2013). Preparedness of Teachers to Maintain Discipline in the Absence of Corporal Punishment in Bondo District, Kenya. Unpublished Master's thesis. Maseno University, Kenya.

Omondi, C. (2010). The Level of Indiscipline after Banning Corporal Punishment in Schools. A Case Study of Ogande Girls High School in Rangwe Division, Homabay District Unpublished P.G.D.E. Research Project). Kenyatta University, Kenya.

Ouma, M.A., Simwata, E.M.W \& Serem, T.D.K. (2013). Management of Pupil Discipline in Kenya: A Case Study of Kisumu Municipality. Available online@http://www. Interesjournals.org.

Owens, R.G. (1987). Organizational Behaviour in Education. New Jersey: Prentice -Hall, Inc.

Owiti, F. (2001, March 21). "Experts give Causes of School Strikes". The Standard, Nairobi: Standard Media Group. . p.17.

Patton, M.O. (1990). Qualitative Evaluation and Research Methods. Newbury Park, London: Sage

Republic of Kenya, (1968). Education Act Chapter 212, (1968) Revised (1980) Laws of Kenya. Nairobi: Government Printer.

Republic of Kenya. (2001a). Report of the Task Force on Students' Discipline and Unrest in Secondary Schools. Nairobi: Ministry of Education, Science and Technology.

Republic of Kenya. (2001b). The Children's Act 2001. Nairobi: Government Printer.

Republic of Kenya. (2013). Education Act. Chapter 212. Nairobi: Government Printer.

Rosen, L (1999). School Discipline: Best Practices for Administrators California: Corwin Press

Samoei, W.K. (2012). The Role of Guidance and Counseling in Management of Student Discipline in Secondary Schools in Londiani District, Kericho County, Kenya .Unpublished Master's thesis. Kenyatta University, Kenya.

Sogoni, N. (1997). Attitudes of Students, Parents and Teachers towards the Use of Corporal Punishment in Senior Secondary Schools. Unpublished Master's thesis. University of Natal, Pietermaritzburg.

Simatwa, E.M.W. (2007). Management of Student Discipline in Secondary Schools in Bungoma District Unpublished Doctor of Philosophy thesis. Maseno University, Kenya.

Simiyu, C.N. (2003). Attitudes of Teachers and Pupils towards Use of Corporal Punishment in Nakuru Municipality Primary Schools, Kenya. Unpublished Master's thesis. University of Kwazulu-Natal, South Africa. Available: http://hdl.handle.net

Smit, E.M. (2010). The Role of School Discipline in Combating Violence in Schools in the East London Region. Unpublished Master's thesis. University of Fort Hare. 
Smith, K. (2008). Learning Styles and Students Perception of Teachers Attitudes Among African American Students in Secondary Education. Unpublished Doctoral dissertation. Louisiana State University, Louisiana.

Strong, T. (2003). Getting Curious About Meaning Making in Counseling. British Journal of Guidance and Counseling 31(3), 259-273.

Thompson, C. (2002, April 24). School crisis of Discipline. The Citizen, p.6.

Waigwa, J.M. (1996). An Investigation into the causes of Indiscipline: The Case of Gathangi Secondary School. Unpublished P.G.D.E. Research Project. Kenyatta University, Kenya.

Wairuri, C. (2004). Teachers Attitude towards the Withdrawal of Corporal Punishment in Public secondary schools in Kikuyu Division. Unpublished Master's thesis. Moi University, Kenya.

Wangari, L.K. (2006).Effectiveness of Guidance and Counseling Program in Combating Indiscipline in Secondary Schools: A case of Nakuru Municipality, Kenya. Unpublished Master's thesis. Egerton University, Kenya.

Yahaya, A. (2009). Disciplinary Problems among Secondary School Students in Johor Bahru, Malaysia. European Journal of Sciences, 4(2), 56-78.

Zulu, B.M., Urbani, G., Van der Merwe, A \& Van der Walt, J.L. (2004). Violence as an impediment to a culture of teaching and learning in some South African Schools. South African Journal of education, 24(2), 170-175.

Cite this Article: Onyango DO, Simatwa EMW, Gogo JO (2016). Influence of Mental Harassment Ban on Student Discipline in Secondary Schools in Kenya: A Case Study of Ugenya, Gem and Siaya Sub-Counties. Greener Journal of Educational Research, 6(3): 133-150, http://doi.org/10.15580/GJER.2016.3.052316095 